\title{
L'épineuse Question De La Place Du Droit Communautaire Au Sein De La Hiérarchie Des Normes Internes : Un Droit Hors Hiérarchie? Réflexion À La Lumière Des Systèmes Constitutionnels Des Etats D'afrique Francophone
}

\author{
Achille Magloire Ngah
}

Docteur/PHD en droit public, Moniteur à la Faculté des Sciences Juridiques et Politiques, Université de Yaoundé II, Cameroun

Doi:10.19044/esj.2019.v15n11p194 URL:http://dx.doi.org/10.19044/esj.2019.v15n11p194

\section{Résumé}

La question de la primauté est une question essentielle dans les rapports de système. En ce sens qu'elle est au cœur de l'effectivité des droits d'origine externes en générale et du droit communautaire en particulier. Au point où la doctrine n'a pas hésité à dire que la primauté était une question existentielle du droit communautaire. Autrement dit, l'ordre juridique communautaire et le doit qu'il secrète ne peuvent se concevoir autrement que primant les ordres juridiques nationaux. Or cette proclamation téléologique de l'ordre communautaire de la vocation de son droit à la primauté n'est pas toujours en phase avec le statut ou la considération que les constitutions se donnent à elles-mêmes. En tant que normes fondant l'ordre juridique national, les constitutions ont tendance à se hisser au sommet de la hiérarchie des normes internes, tout en ne permettant au droit externe (international et communautaire) qu'une place supra législative. Ce contraste mettant côte à côte deux ordres juridiques, prônant chacun sa suprématie mérite de retenir notre attention dans un continent comme l'Afrique qui fait de l'intégration régionale une nécessité à l'atteinte de ses objectifs. Il est question d'analyser le traitement que les ordres constitutionnels offrent au droit communautaire d'une part, et l'incidence de l'intégration régional sur la hiérarchie des normes internes des Etat. Pour y parvenir, l'étude comparée des systèmes constitutionnels d'Afrique francophone, aux moyens d'un positivisme sociologique, nous a permis de constater que le droit communautaire est un droit hors hiérarchie, sa place au sein de la hiérarchie classique interne est encore à déterminer 
Mots Clés : $\quad$ Ordre juridique communautaire, ordre constitutionnel/constitution, hiérarchie des normes, primauté, rapports entre les ordres/ systèmes juridiques

\title{
The Thorny Question of the Place of Community Law in the Hierarchy of Internal Standards: A Non- Hierarchical Right? Reflection in the Light of the Constitutional Systems of French-Speaking African States
}

\author{
Achille Magloire Ngah \\ Docteur/PHD en droit public, Moniteur à la Faculté des Sciences Juridiques \\ et Politiques, Université de Yaoundé II, Cameroun
}

\begin{abstract}
The issue of primacy is a key issue in system reports. In the sense that it is at the heart of the effectiveness of external rights in general and of Community law in particular. At the point where the doctrine did not hesitate to say that primacy was an existential question of community law. In other words, the Community legal order and the secret order must not be conceived otherwise than taking precedence over the national legal orders. But this teleological proclamation of the community order of the vocation of its right to primacy is not always in phase with the status or the consideration that the constitutions give themselves. As constitutions of the national legal order, constitutions tend to rise to the top of the hierarchy of internal standards, while allowing the external law (international and community) only a supralegislative place. This contrast between two legal orders, each advocating its supremacy deserves our attention in a continent like Africa that makes regional integration a necessity to achieve its objectives. It is a question of analyzing the treatment that constitutional orders offer to community law as a whole on the one hand, and the impact of regional integration on constitutional orders on the other hand. To achieve this, the comparative study of the constitutional systems of Africa, through the means of a sociological positivism, has allowed us to observe that Community law is a non-hierarchical right, its place in the internal hierarchy is still to be determined.
\end{abstract}


Keywords: Community legal order, constitutional order/constitution, hierarchy of internal standards, primacy, relationship between legal orders or law systems

\section{Introduction:}

« Condition existentielle du droit communautaire » (PESCATORE P., 2005, p. 85), le principe de primauté est d'une importance capitale pour une étude portant sur les rapports ordres juridiques communautaire et constitutionnel. En effet, c'est grâce à ce principe de primauté que la normativité du droit communautaire prend tout son sens (PICOD (F.), 2007). Étant entendu que la primauté reflète la spécificité de l'ordre juridique communautaire ainsi que la nécessité de garantir son autonomie, son autorité et son efficacité par rapport au droit interne des États membres et singulièrement au droit constitutionnel. Dans l'ordre international et communautaire, la question de la primauté de ces ordres sur les ordres internes ne semble souffrir d'aucun doute. Les articles 26 et 27 de la Convention de Vienne du 23 mai 2019 sur le droit des traités illustrent la position du droit international. Les ordres juridiques communautaires quant à eux prévoient expressément la primauté interne du droit sécrété par leurs institutions. En effet, il ressort de la lecture de l'article 44 du traité CEMAC version de 2008 et 2009 que «les actes adoptés par les Institutions, Organes et Institutions Spécialisées de la Communauté pour la réalisation des objectifs du présent Traité sont appliqués dans chaque État membre nonobstant toute législation nationale contraire, antérieure ou postérieure. » De l'avis de Jacques FIPA NGUEPJO, « cette prééminence est inconditionnelle dans la mesure où le droit communautaire ne tire pas sa suprématie d'une quelconque concession de la part du droit des États mais se fonde sur sa nature intrinsèque » (FIPA NGUEPJO (J.), 2011, p. 39). Mais cette posture téléologique et objectiviste du droit communautaire doit être nuancée, car aucun État, par le biais de sa constitution, ne peut se voir imposer un « droit d'origine externe » (ONDOUA (A.), 2014. P.296 ) contre sa volonté (KAMTO (M.), 2007). En effet, il parait de plus en plus évident que «la primauté interne du droit communautaire semble ne pas traduire autre chose que l'expression de la volonté des parties contractantes » (BRIBOSIA (H.), 1996p.57). C'est ce qu'exprime le Professeur Alain PELLET lorsqu'il laisse entendre que le Traité constitutif d'une organisation d'intégration est avant tout « ...un traité » (PELLET (A.), 1994, p. 264) et comme tel, il est « ancré » dans les ordres constitutionnels des États membres. De par ces dernières considérations, il sera question dans cette contribution de s'interroger sur l'autorité du droit communautaire et de son ordre juridique dans les constitutions africaines. Ceci, à travers un essai

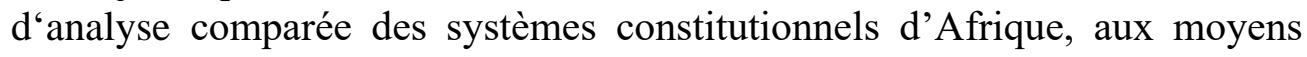


d'un positivisme à la fois juridique et sociologique pour rendre compte de l'état actuel des choses.

Dans une perspective purement dualiste, cette question n'aurait pas de raison d'être, en ce sens que les ordres juridiques vivraient séparément et indépendamment sans imbrication. Mais les États d'Afrique francophone, que nous étudions, étant de culture moniste, et surtout que l'ordre juridique communautaire postulant le monisme, et considérant la « jalousie » viscérale des États africains pour la préservation de la souveraineté de leurs États, il n'est guère surprenant que le constituant africain ait érigé des garde-fous contre les débordements normatifs des ordres internationaux (I). Cependant, les autorités étatiques, qu'elles soient exécutives, législatives et judiciaires ayant la lourde charge d'appliquer à la fois le droit national dont ils tirent les titres de compétence de la constitution et le droit communautaire dont la primauté a été « consentie » (DUBOUT (E.), 2005) par la même constitution, sont interpellées au premier chef pour ménager ces deux titres de compétences. Chose qui n'est guère aisée pour ces autorités (II), en ce sens que l'avènement de l'ordre juridique communautaire a opéré une véritable transformation de leur rôle.

\section{I- L'ENCADREMENT CONSTITUTIONNEL DE LA PRIMAUTE DU DROIT COMMUNAUTAIRE}

La lecture attentive des constitutions d'Afrique noire francophone révèle que ces États n'ont pas que la langue française en commun. En effet, les règles de conflit, telles qu'énoncées aux articles 54 et 55 de la Constitution française - «Si le Conseil constitutionnel, saisi par le président de la République, par le premier ministre, par le président de l'une ou l'autre assemblée ou par soixante députés ou soixante sénateurs, a déclaré qu'un engagement international comporte une clause contraire à la Constitution, l'autorisation de ratifier ou d'approuver l'engagement international en cause ne peut intervenir qu'après la révision de la Constitution. »: article 54 et 55 «Les traités ou accords régulièrement ratifiés ou approuvés ont, dès leur publication, une autorité supérieure à celle des lois, sous réserve, pour chaque accord ou traité, de son application par l'autre partie. » - sont la référence unanimement partagée par les lois fondamentales soumises à notre étude. Ces dispositions consacrent tour à tour, la suprématie de la constitution sur tous les traités et accords internationaux (B) d'une part et la primauté du droit d'origine externe sur les lois et règlements internes d'autre part $(\mathrm{A})$. 


\section{A- La primauté incontestable de la constitution sur toute les normes externes et internes}

Comme cela vient d'être énoncé ci-dessus, les constituants africains ont clairement repris en chœur les termes de l'article 55 de la constitution française.(A). Ceci entraine que la primauté du droit communautaire dans les ordres juridiques constitutionnels internes des États n'est pas différente, mieux, se confond avec celle dont bénéficie le droit international. (B)

\section{1- L'existence d'une règle de conflit quasi identique de primauté, plagiat de} l'article 55 de la Constitution française

Les constituants africains ont clairement opté pour la primauté de l'ordre juridique international sur toute la législation nationale inférieure à la constitution. Ce qui entraine que ces «textes constitutionnels modernes s'inspirent d'une conception nettement moniste des rapports entre le droit international et le droit interne » (DE VISSCHER (P.), 1951, p. 516), mais avec suprématie de la constitution sur le droit d'origine externe (droits international et communautaire).

Quelques exemples de cet état des choses : la Constitution béninoise, en son article 147, énonce que "les traités ou accords régulièrement ratifiés ont, dès leur publication, une autorité supérieure à celle des lois, sous réserve, pour chaque accord ou traité, de son application par l'autre partie. " L'article 223 (ancien 184) de la Constitution congolaise n'en dit pas autre chose, puisqu'il affirme que " les traités ou les accords, régulièrement ratifiés ou approuvés, ont dès leur publication, une autorité supérieure à celle des lois sous réserve, pour chaque accord ou traité, de son application par l'autre partie ». Le même principe est consacré par l'article 98 de la Constitution sénégalaise. Signalons aussi que la Constitution du Cameroun est dans la même veine en son article 45.

Manifestement, la règle de conflit établie par l'article 55 de la Constitution Française de 1958 a énormément séduit le Constituant africain d'expression française. Cela est d'ailleurs confirmé même au-delà des anciennes zones d'influence française, avec notamment le cas de la RDC dont la Constitution consacre la même disposition à son article 215 avec cependant une légère modification des expressions « ratifiés ou approuvés », remplacées par « conclus ». Ce pendant, de toutes ces constitutions, aucune ne fait de distinction entre le droit international général et le droit communautaire.

\section{2- La non spécificité de la Primauté du droit communautaire : une primauté relativement identique à celle du droit international}

D'après un auteur contemporain, l'ordre juridique communautaire bénéficie d'une hiérarchisation plus forte que bien des États à structure fédérale ou régionale, en ce sens que, selon le même auteur, « la spécificité de 
la primauté communautaire consiste par conséquent en ce qu'elle est ellemême immédiate dans certains cas...[car], ce sont les normes communautaires directement applicables qui prévalent en tant que telles sur les normes nationales contraires...Toute véritable application directe d'une norme suppos[ant] qu'en effet il n'y ait pas d'autre norme contraire dont l'application directe est également possible et qui soit plus forte. » (PFERSMANN (O.), 2005). Cette affirmation, bien qu'excessive, témoigne du «fantasme » qui anime une frange importante de la doctrine, à la suite de la Cour de Justice de l'Union Européenne (CJCE, 22 juin 1965, San Michele, aff. 9/65, Rec. 1967, 35 ; CJCE, 17 décembre 1970, Internationale Handelsgesellschaft, aff. 11/70, Rec. 533), de voir le droit communautaire primer absolument sur les droits nationaux. Seulement, les constituants des États d'Afrique francophone, à l'exemple de leur homologue français, n'ont fait aucune distinction entre l'ordre juridique international et l'ordre juridique communautaire. En effet, toutes les dispositions ci-dessus citées parlent des «traités ou accords internationaux régulièrement approuvés ou ratifiés ». Autrement dit, cette règle de "primauté contingente » (BERRAMDANE (A.), 2002, p. 216) du droit international sur la loi doit être prise au sens large, c'est-à-dire, tout le droit d'origine externe provenant de toutes les conventions internationales auxquelles les États concernés sont parties. Cependant, non seulement cette solution classique de primauté ne satisfait pas les prétentions à la spécificité du droit communautaire, dont la primauté «s'avère absolue car elle s'exerce également à l'encontre des normes constitutionnelles »(ONDOUA (A.), 2001), mais aussi la lecture intégrale de ces dispositions inspirées par l'article 55 de la constitution française révèle ça et là une condition de réciprocité qui, de toute évidence, ne saurait s'appliquer sur les conventions multilatérales et les conventions de droit communautaire. Cependant, la plus remarquable est la condition de conformité de l'engagement externe à la constitution.

\section{B- La primauté du droit communautaire conditionnée par la suprématie de la constitution}

En dépit de la règle de conflit ci-dessus étudiée et qui reconnait au droit externe une valeur supra législative, une autre disposition de la constitution française a fortement séduit le constituant d'Afrique francophone: il s'agit de l'article 54. D'après cette disposition constitutionnelle, «Si le Conseil constitutionnel, saisi par le président de la République, par le Premier Ministre, par le Président de l'une ou l'autre assemblée ou par soixante députés ou soixante sénateurs, a déclaré qu'un engagement international comporte une clause contraire à la Constitution, l'autorisation de ratifier ou d'approuver l'engagement international en cause ne peut intervenir qu'après la révision de la Constitution. » (Article 54 de la Constitution française du 04 octobre 1958) Le succès de cette disposition tient 
du fait qu'elle est manifeste d'un réel « souci de préserver ce qui subsiste de la souveraineté et du domaine » de l'ordre juridique constitutionnel, notamment de sa suprématie(A). Suprématie que les juges, le juge constitutionnel en tête, s'activent à protéger (B).

\section{1- Des constitutions " maîtres du jeu " de leur primauté}

Avant de procéder l'analyse des dispositions constitutionnelles africaines qui traduisent cette filiation ostensible avec la constitution française (b), il est utile de s'appesantir sur la controverse que suscite cette disposition, notamment celle de savoir quelle primauté est consacrée, celle de la constitution ou celle des droits extérieurs (a).

\section{a- La controverse autour de l'article 54}

D'après un auteur contemporain, «tout l'intérêt du monisme théorique consiste en effet à établir le lien entre la question de la hiérarchie des normes et celle des rapports de systèmes. » (PFERSMANN (O.), 2005, pp. 138-140). En d'autres termes, l'articulation des normes est au cœur des rapports qui s'établissent entre l'ordre juridique interne et les ordres externes. Et vu que chaque ordre juridique postule sa suprématie ou son indépendance ou encore son autonomie, il ressort de part et d'autre des règles devant assurer cette suprématie. Tel est le cas dans les ordres constitutionnels des normes de référence de contrôle de constitutionnalité. Ce sont des normes « filtres » de la pénétration du droit externe en droit interne. L'article 54 de la constitution française, repris en boucle par les constituants d'Afrique francophone, est la norme de référence par excellence élaborée pour consacrer la suprématie de la constitution sur toutes les autres normes. Cependant, les choses ne paraissent pas si simples et automatiques, car du fait du caractère imprécis et limité de cette disposition, elle a de tout temps nourri une controverse sur le degré de primauté qu'elle consacre.

D'aucuns ont laissé entendre qu'elle consacrait la supériorité du droit externe sur la constitution, en ce sens que, en cas de contradiction entre une norme d'origine externe et la constitution, c'est cette dernière qui cède pour laisser la place à la norme externe. Par conséquent, pour les tenants de ce courant, cette amovibilité ne peut être comprise autrement qu'en termes d'une part de reconnaissance par la constitution elle-même, de sa soumission au droit externe et d'autre part d'expression de sa révérence ou de sa déférence, car c'est la norme inférieure qui s'efface devant la norme supérieure. Comme le remarque le Professeur Alain ONDOUA, « ces auteurs mettent en avant le fait qu'en cas de contrariété d'un engagement international avec la constitution, c'est à cette dernière de s'adapter au premier par le biais d'une révision constitutionnelle. » (ONDOUA (A.), 2001, p154) Confirmant de ce fait «la 
mise au pas de la constitution » (idem) qui ne saurait traduire autre chose que la manifestation de sa soumission au droit d'origine externe.

Bien que tout cela semble logique et même en phase avec l'idéal du maître autrichien KELSEN, parrain de la théorie de la hiérarchie des normes et surtout de l'unité de l'ordre juridique avec suprématie de l'ordre international ou des ordres externes, cette lecture «internationaliste » et « communautariste » des rapports droit interne/droit externe pèche par son infidélité au dogme kelsenien de la source de la norme inférieure. En effet, dans la théorie de KELSEN, chaque norme tire sa source de la norme supérieure, ce qui induit que la constitution devrait tirer sa source du droit externe. Chose pas aisée à démontrer, en dehors des cas d «'internationalisation des constitutions des États en crise» qui apparaissent comme un « phénomène d'aspiration des compétences et des matières constitutionnelles étatiques vers l'extérieur, traduit par un double mouvement, celui du droit international vers les ordres juridiques des États et celui inverse des constitutions étatiques vers l'ordre juridique international. Elle est donc ou volontaire ou contrainte, mais toujours rattachée à des circonstances précises qui en déterminent le contenu et, surtout, le degré » (NDJIMBA (K.F.), 2011, 663 P., préc.17). Il n'est pas évident de démontrer que la constitution tire sa source du droit externe, ce d'autant plus qu'il ressort des développements cidessus présentés que l'engagement des États dans les ordres juridiques externes est un acte de volonté consacré par les constitutions des États. La constitution, tout compte fait, demeure au centre de la détermination du destin et du droit d'origine externe et du sien elle-même.

Avant d'aborder le courant des tenants de la supériorité de la constitution, il existe un courant intermédiaire que le Professeur BERRAMDANE qualifie des adeptes du «ni-ni ». Pour ses défenseurs (BECHILLON (D.), 1996 ; LUCHAIRE (F.), 1992), la règle de l'article 54 de la constitution française, reprise en chœur par les constitutions des États d'Afrique francophone, ne consacre ni la primauté du droit externe, ni la primauté de la constitution. Ils estiment que «[c]e texte constitutionnel n'apporte pas d'éclairage très précis sur les rapports constitution-droit international » (RIDEAU (J.), 1990, p.270.). Tout au plus, « il prévoit tout simplement une procédure plus lourde de ratification des traités ».

S'agissant des partisans de la primauté absolue de la constitution sur le droit d'origine externe, cette doctrine soutient que, bien que 1'article 54 de la constitution française et ses doublons contenus dans les constitutions de l'Afrique francophone promeuvent le monisme, ce dernier « cède devant la suprématie constitutionnelle » (GREWE (C.)-RUIZ FABRI (H), cité par ONDOUA (A.), op cit. 152) . Tout leur raisonnement est endossé sur le caractère facultatif de la saisine de la juridiction constitutionnelle et de la révision préalable de la constitution avant la ratification ou l'approbation d'un 
engagement international. Autrement dit, l'intervention du pouvoir constituant, en cas de contrariété d'un engagement international avec la constitution, n'étant pas une obligation, mais une simple faculté laissée à l'appréciation des autorités habilitées à saisir le juge constitutionnel, il est envisageable qu'un engagement inconstitutionnel ne saurait s'imposer de force dans l'ordre juridique interne, ou du moins il ne saurait produire des effets juridiques sans l'accord manifeste du constituant à travers la révision de la loi fondamentale. Si cette lecture peut être nuancée, notamment vis-à-vis du droit communautaire dérivé qui, en principe, bénéficie indubitablement des caractères d'effet direct et d'effet immédiat, il n'en demeure pas moins vrai que l'article 54 de la constitution française et ses doublons africains, traduisent un souci avoué de garantir l'autonomie et partant, la suprématie de la constitution, considérée comme le texte qui attribue valeur et portée aux normes juridiques qui lui sont inférieures, entre autres, les traités et accords internationaux.

\section{b- Une disposition filtre pour la préservation de la souveraineté des États}

D'emblée, relevons que la technique ou le principe de contrôle de constitutionnalité a priori que consacrent les dispositions constitutionnelles ci-dessus présentées n'a pas pour but premier la garantie ou la préservation de la hiérarchie des normes, mais plutôt l'évaluation de la conformité ou de la compatibilité de la norme d'origine externe à la constitution. Et comme nous l'avons souligné plus haut, en dépit de l'imprécision qui ressort de la lecture de ces dispositions à l'aune de la théorie de la hiérarchie des normes, nous pouvons soutenir, en nous basant sur la technique juridique, que les constitutions entendent par ce contrôle, préserver leur autonomie ou la souveraineté des États. En effet nous savons que l'office du juge constitutionnel en matière de contrôle de constitutionnalité des engagements internationaux n'est pas de «censurer » l'engagement international, mais plutôt de vérifier si ce dernier est compatible avec la constitution. Dans cette vérification, il lui est curieusement interdit d'interpréter l'engagement international alors qu'il est appelé à déterminer la validité du texte en droit interne. Dans cette situation, peut-on valablement déterminer la validité d'un texte sans le censurer le cas échéant?

C'est à ce dilemme qu'a été confronté le Conseil constitutionnel français lorsqu'il lui était posé la question en 2004 (CC, Décision n ${ }^{\circ}$ 2004-505 DC du 19 novembre 2004) de savoir si la règle de primauté consacrée par l'article I-6 du traité établissant une constitution pour l'Europe, était compatible à la constitution. De l'avis de la doctrine, la réponse positive donnée par la Haute juridiction française ne va pas de soi, en ce sens que celleci aurait outrepassé ses compétences en interprétant le texte communautaire lui-même. Pour Mme Samira BOUSSETTA, «On peut penser que si le 
Conseil constitutionnel élargit ses compétences, ce n'est pas pour opérer un contrôle de hiérarchie des normes [...], mais pour interpréter une norme internationale afin d'en proposer une lecture qui permet sa compatibilité avec son interprétation jurisprudentielle des dispositions constitutionnelles» (BOUSSETTA (S.), 2017), le but dans cet exercice étant de préserver « le règne de la constitution » (CAMBY (J.P.), 2004, n 4, p. 888.). Ici tout repose sur «la logique juridique et fait référence à la notion de consentement souverain» (DUBOUT (E.), «De la primauté « imposée » à la primauté « consentie »..., op cit.). En effet, comme le démontre parfaitement $M$. DUBOUT, «L'antériorité de l'ordre juridique interne par rapport à tout ordre juridique d'origine conventionnelle serait ainsi le fondement de l'incontournable supériorité du premier sur le second, qui même en se subordonnant présuppose sa suprématie sur ce à quoi il se soumet. En ce sens, le consentement à la primauté ne pourrait pas être véritablement entier, sauf à basculer dans un système fédéral : une Constitution ne peut renoncer à sa qualité de norme suprême sans perdre du même coup son titre constitutionnel »

On le voit bien, nonobstant l'existence d'une disposition consacrant la spécificité et même, pourrait-on dire, la primauté du droit communautaire, le juge constitutionnel français conditionne la suprématie du droit communautaire à celle de la constitution. Qu'en est-il de ses confrères africains?

\section{2- Des juges jouant le jeu de la constitution}

Aujourd'hui en Afrique, « il est indéniable que le droit international de façon général et le droit communautaire de façon spécifique occupent une place de plus en plus importante parmi les normes applicables à l'intérieur des États » (SAWADOGO (M.F.), 2003, p.77.). Cette situation met le juge au cœur de l'ordonnancement des ordres juridiques surtout lorsqu'il s'agit de faire prévaloir, en cas de conflit, une norme -soit interne, soit communautaire ou internationale- sur l'autre. Ce qui est loin d'être une tâche facile, tant il est vrai que non seulement chaque ordre juridique postule sa suprématie, mais le juge interne, et surtout constitutionnel des États monistes de l'Afrique francophone qui s'abreuvent sur la tradition constitutionnelle française, vivent un dilemme : protéger la suprématie de la constitution d'une part et assurer l'intégration des États dans les ordres juridiques externes auxquels ils ont consenti d'autre part.

Quoiqu'il en soit, face à cette montée en puissance du droit d'origine externe en général et du droit communautaire en particulier, il est incontestable que la protection juridictionnelle de la constitution constitue aujourd'hui la meilleure garantie de la suprématie de la constitution dans l'ordonnancement juridique interne des États (OUEDRAOGO (S.M.), 2011, 
p.194). Nonobstant la rareté et la timidité des marques juridictionnelles africaines, soulignons d'emblée, avec le Pr. Marcelin NGUELE ABADA, que l'existence dans les constitutions des États d'Afrique noire francophone du modèle préventif du contrôle de constitutionnalité est une conséquence logique, tout au moins une condition de la suprématie de la constitution (NGUELE ABADA (M.), 2007, p.45 ). Sur la base de ce principe, on peut donc dire que le juge constitutionnel a le monopole de la protection de la constitution contre toutes les atteintes des ordres juridiques externes.

Dans cette optique, le juge constitutionnel béninois, saisi sur la base de l'article 146 de la constitution, a eu à se prononcer sur la constitutionnalité du traité OHADA. A travers une décision de principe du 30 juin 1994 (Décision DCC 19-94 du 30 juin 1994), les juges de la Haute juridiction du Benin ont réussi, comme leurs homologues français, à concilier suprématie de la constitution et objectif de l'intégration régionale ou africaine. En effet, pour la Cour, tout engagement international, quel qu'il soit et ceux relatifs à l'intégration régionale ou sous régionale en particulier entraine abandon partiel de souveraineté consenti par les États. De ce fait, poursuit-elle cet «abandon partiel de souveraineté dans le cadre d'un traité, ne saurait constituer une violation de la Constitution, dans la mesure où celle-ci, en disposant dans son article 144 que le Président de la République négocie et ratifie les traités et accords internationaux, et ce dans tous les domaines, a prévu la possibilité de conclure des traités ». Par conséquent, les Juges vont conclure à la compatibilité du traité OHADA à la Constitution béninoise en fondant l'engagement communautaire de l'État béninois sur la constitution elle-même. Ainsi, peut on lire dans les conclusions de l'arrêt, qu'étant donné que ledit Traité " contient [...] un abandon partiel de souveraineté, dans le but affirmé par le préambule 'd'accomplir de nouveaux progrès sur la voie de l'Unité Africaine',' ") et l'article 149 de la Constitution, aucune clause de ce traité n'a été jugé contraire à la constitution.

Dans la même donne tendant à rechercher la conciliation entre la prééminence de la constitution et l'engagement communautaire, le juge constitutionnel sénégalais proclame la constitutionnalité du traité OHADA en justifiant le fondement de l'engagement de l'État du Sénégal dans la constitution. Pour le juge sénégalais, " considérant en effet que la réalisation de l'Unité africaine impliquant nécessairement un abandon de souveraineté de la part des États qui y participent, le peuple sénégalais, par cette décision constitutionnelle, accepte d'accomplir un tel effort; qu'il s'ensuit qu'un engagement international par lequel le Sénégal consentirait à abandonner sa souveraineté dans ce but serait conforme à la constitution » (Décision

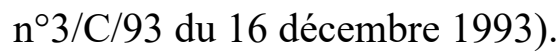

Il n'est pas superfétatoire de rappeler ici l'avis de la Cour Suprême congolaise qui, bien que fortement contesté, témoigne de la prégnance de la 
constitution dans la hiérarchie des normes internes en Afrique noire francophone. Dans cet avis, le juge suprême congolais fait de la souveraineté et de l'indépendance du Congo, le seuil infranchissable par tout accord international. Ainsi, l'on peut lire dans l'argumentation du juge que «la fonction de juger [...] est une fonction constitutionnelle en même temps qu'elle est l'expression de la souveraineté et de l'indépendance nationale »; aussi, poursuit la Cour, "le pouvoir de rendre exécutoire sur le territoire national une décision juridictionnelle rendue par une juridiction étrangère ou une sentence arbitrale appartient aux seules juridictions nationales et procède également de la souveraineté et de l'indépendance nationale ». (CS (Congo), Avis du $1^{\mathrm{er}}$ octobre 1998)

Ces quelques cas de contrôle de constitutionnalité témoignent de l'option souverainiste du juge constitutionnel d'Afrique francophone. Pour lui, la constitution est incontestablement la norme suprême, en ce sens que c'est elle qui fonde la validité des autres normes au sein de l'ordre juridique interne. Certes, ces décisions présentent une vision, mais ne suffisent pas pour rendre compte de toute la problématique des rapports de système entre l'ordre juridique interne et les ordres juridiques externes, surtout l'ordre communautaire au regard de la force de pénétration des normes communautaires dans l'ordre interne.

\section{II- LES FONCTIONS DE L'ETAT A L'EPREUVE DE LA PRIMAUTE DU DROIT COMMUNAUTAIRE}

L'État est une entité ambivalente surtout dans l'exercice de sa souveraineté. Il peut prétendre à la fois à la souveraineté et à l'appartenance à une organisation d'intégration. Dans ces deux cas, on parlera d'exercice de la souveraineté interne et internationale. Les deux qualités sont compatibles (BÉLIGH NABLI, 2007, P.117) et appellent de la part des autorités publiques de chaque État « un dédoublement fonctionnel», car elles sont appelées à appliquer à la fois le droit communautaire et le droit national, en ce sens que, pour les institutions communautaires et pour l'ordre juridique qu'elles incarnent, les États membres et leurs autorités sont «des instruments d'intégration » (HECQUARD-THERON (M.), 1990, n 4, p. 693-711, spéc. p. 698). Tout se passe comme si, en dépit de la primauté consacré de la Constitution sur toutes les normes externes, chaque État doit se débrouiller à appliquer le droit communautaire. Cependant, de façon générale, il apparait une mise à l'épreuve de la répartition classique des compétences au sommet des États (A), tandis que la fonction de juger est réévaluée ou revalorisée de par sa communautarisation (B). 


\section{A- La répartition classique des compétences éprouvée lors de l'élaboration et de l'application du droit communautaire}

Les lignes qui suivent vont nous permettre de démontrer que le pouvoir législatif «dispose d'un rôle relativement modeste » (BÉLIGH NABLI «L'Union des États et les États de l'Union», op cit. p.100) dans l'élaboration du droit communautaire, au point où l'on parlerait de dépossession du parlement de ses compétences législatives. Cette dépossession des parlements nationaux de leurs compétences législatives est manifeste à tous les niveaux de la vie des normes communautaires. On le constate lors de l'élaboration du droit communautaire primaire, au regard des seules constitutions, et de celle du droit dérivé, si l'on observe les procédures d'élaboration en vigueur au sein de certaines organisations telles que la CEMAC et de la CEDEAO.

Le pouvoir exécutif a le privilège, voire l'exclusivité de l'élaboration du droit communautaire originaire. En effet, de la lecture des constitutions de certains États, le gouvernement, plus précisément le Chef de l'État, est maître de la conduite des relations internationales. De ce fait, la négociation et la conclusion des traités internationaux en général, et ceux créant les organisations de droit communautaires en particulier, relèvent de sa compétence, tandis que «les parlements nationaux se bornent à ratifier les Traités, sans possibilité d'amender ce qui relève du privilège exécutif de la négociation diplomatique »(DE BERRANGER (T.), 1995, p. 366) plus connu en droit international sous le vocable treaty-making power.

Pour ce qui est de l'élaboration du droit dérivé, il ressort que bien que clamant et réclamant le dépassement de l' «interétatisme » au profit de l'intégration des souverainetés, ou de la gestion en commun de ces souverainetés, les organisations d'intégration régionale ne sauraient se départir totalement de « la dérive intergouvernementale» (DE BERRANGER (T.), 1995, p. 358) qui est au cœur de toutes les organisations internationales qui, rappelons-le, sont, d'abord et avant tout, des tribunes intergouvernementales. Par conséquent, l'élaboration de la norme communautaire dérivée se présente davantage comme le produit d'une négociation diplomatique. Ainsi le mode d'élaboration de la législation communautaire met en scène les représentants des exécutifs nationaux, tant en amont au stade de sa préparation, qu'en aval au stade de sa discussion et de son adoption.

$\mathrm{Au}$ niveau des États, chaque gouvernement prévoit des administrations chargées de la coordination et du suivi de la politique communautaire de l'État. Ces ministères qui peuvent avoir divers dénomination ou diverses charges connexes sont le plus souvent soit des départements ministériels en charge des relations internationales, avec en leur sein des directions chargées de l'intégration régionale ; soit des départements 
ministériels exclusivement consacrés aux questions de l'intégration africaine et/ou régionale ou encore des ministères chargés des questions économiques et de la planification. Ces structures administratives se démarquent comme de véritables interfaces entre les Etats membres et les organisations d'intégration régionale. Devenant ainsi les portes voix des Etats d'une part et les serviteurs des ordres juridiques communautaires d'autre part.

De ce qui précède, le premier constat est l'exclusion des parlements nationaux lors de la mise sur pied des stratégies nationales pouvant aboutir à (ou influencer) l'élaboration de la législation communautaire.

De manière générale, il ressort du droit institutionnel communautaire que les institutions ou organes qui exercent la fonction législative sont le plus souvent constitués, ou presque toujours, de représentants directs des exécutifs nationaux. Cela traduit certainement l'expression d'un souci d'efficacité dans la conduite des objectifs communs fixés par les Etats, car, la maitrise des relations diplomatiques étant du ressort de l'exécutif, qui d'autre, mieux que ses agents, pourrait mieux assurer la concrétisation normative desdits objectifs? C'est dans cet esprit que le chapitre 3 du traité révisé CEMAC de 2009, consacré aux actes juridiques de l'organisation et au contrôle de ses activités, énonce à l'article 40 que la Conférence des Chefs d'État adopte des actes additionnels au Traité et prend des décisions ; le Conseil des Ministres et le Comité Ministériel adoptent des règlements, règlements cadres, des directives, prennent des décisions et formulent des recommandations ou des avis ; les premiers responsables des Institutions, Organes et Institutions Spécialisées de la Communauté arrêtent des règlements d'application, prennent des décisions et formulent des recommandations ou des avis. L'article 12 du traité CEDEAO stipule, quant à lui, que les actes du Conseil sont dénommés règlements.

L'une des critiques, formulées par une certaine doctrine " communautaro-sceptique " (L'expression est de nous, notamment en analogie avec des concepts tels que « euro sceptique » ou « afro sceptique »), est le " déficit démocratique » dans le processus institutionnel et décisionnel communautaire (DE BERRANGER (T.), 1995, p.342), de sorte qu'il revient toujours la question de savoir si les limitations de souveraineté consenties par les États au profit de la communauté sont exercées à due concurrence par les autorités communautaires équivalentes. En d'autres termes, l'élaboration de la norme communautaire respecte-t-elle toutes les exigences démocratiques qui gouvernent les États modernes, notamment : le respect des droits et libertés de la personne humaine, la prise des décisions par des représentants démocratiquement élus, la séparation des pouvoirs...?

L'examen du processus d'élaboration et d'adoption de la norme communautaire- tant dans le cadre de la CEMAC et de la CEDEAO que de l'Union Européenne- montre que « la démocratie au sein [des organisations 
d'intégration] n'est pas une affaire de vases communicants » (SUBTIL (J.B.), 1990). En effet, les compétences parlementaires nationales ont été transférées aux représentants, mieux, aux agents des exécutifs nationaux, qui trônent dans les institutions communautaires, de sorte que le législateur communautaire n'est, à la vérité, qu' "une conférence diplomatique secrète » (DE BERRANGER (T.), 1995, p. 345). La norme communautaire n'est pas l'émanation de la volonté directe du peuple, « source vive du pouvoir dans les démocraties » modernes. Elle émane plutôt du Conseil des Ministres ou du Comité Ministériel qui n'est rien d'autre qu'un organe composé des représentants des exécutifs nationaux. Cela n'est guère une surprise compte tenu de l'omnipotence des pouvoirs exécutifs sur le plan national.

En effet, l'observation des régimes politiques africains fait apparaitre des chefs d'États et des gouvernements si forts que ces hommes d'État finissent par créer une confusion entre leur personne et l'État. Au regard de ce qui précède les exécutifs nationaux semblent être les maîtres à penser et à exécuter le droit communautaire au détriment des parlements nationaux. Tout comme l'on assiste aussi à une transformation des compétences du juge national.

\section{B- La réévaluation des missions du juge national}

Le principe de l'immédiateté du droit communautaire entraine que ce dernier, dès son entrée en vigueur constitue le droit positif de chaque État membre, et par ce fait, le juge national a l'obligation de l'appliquer (1) ; en cas de doute ou de flou, il doit se référer à la juridiction communautaire pour requérir son interprétation (2)

\section{1- Le juge national : juge communautaire de droit commun}

Le processus d'intégration régionale et l'intégration juridique inhérente à la construction communautaire modifient substantiellement la dimension institutionnelle de l'office du juge interne (CANIVET (G.), 1992, p. 143). Les tribunaux internes, qui ont leur compétence déterminée par le droit national, doivent en l'espèce statuer en fonction du droit communautaire (BATAILLER (F.), 1963. p.743). « La compétence du juge national en matière $\mathrm{d}$ 'application du droit communautaire repose en principe sur la conception que celui-ci fait partie intégrante du droit applicable sur le territoire des États membres » (GNIMPIEBA TONNANG (E.),NGUENA DJOUFACK (A.L.), 2014). Cette ambivalence fonctionnelle place le juge national au cœur de l'articulation de l'ordre juridique communautaire avec l'ordre juridique de l'État. Ce juge devra tenir compte d'une combinaison de règles et de techniques, les unes ressortissant au droit interne avec lesquelles il est familiarisé, les autres relevant du droit communautaire avec lesquelles il commence à se familiariser. Toutes choses qui font du 
juge interne, selon une expression désormais consacrée, «le juge communautaire de droit commun », en ce sens que de par ses soins, les citoyens, mieux les particuliers de chaque État membre d'une organisation d'intégration, tant dans leurs relations entre eux (relations horizontales), que dans leurs relations avec l'État dont ils relèvent (relations verticales), se voient directement appliquer le droit communautaire sans que des procédures ou lois de réceptions soient nécessaires.

En effet, l'ordre juridique communautaire a renvoyé « explicitement au juge national le devoir d'assurer la sanction de son droit. Ce faisant, celuici est devenu le premier garant jurisprudentiel du respect de ce dernier » (GNIMPIEBA TONNANG (E.),NGUENA DJOUFACK (A.L.), 2014) C'est l'expression des principes d'effet direct et immédiat du droit communautaire qui ont clairement « communautarisé » l'office du juge interne et ont fait de ce dernier une sentinelle de la primauté du droit communautaire au sein des ordre juridiques internes. Ce rôle de veilleur du respect du sacro-saint principe de primauté du droit communautaire sur tout le droit national, «fait obligation au juge national d'appliquer intégralement le droit communautaire, en faisant de sa propre autorité tout ce qui est nécessaire pour écarter une disposition interne contraire, nonobstant tout obstacle législatif, administratif ou judiciaire» (CANIVET (G.), 1992, p. 144-145) même postérieur à ce droit commun. C'est la non pertinence de la maxime lex postérior derogat priori en matière de confrontation entre le droit communautaire et le droit interne par les juges internes. Seul doit primer le droit communautaire. La mise en œuvre de cette " condition existentielle » dévoie le titre de compétence originelle du juge national, serviteur de la Constitution, pour faire de lui un serviteur du droit communautaire.

\section{2- Le juge national : cheville ouvrière de l'application, donc de l'interprétation du droit communautaire}

En règle générale, il est reconnu au juge interne la compétence d'application et d'interprétation des règles du droit international dans la mesure où ces règles sont nécessaires pour solutionner un différend porté devant lui. La Cour fédérale de justice du Cameroun a eu à le relever en soulignant que « les conventions internationales constituent des sources du droit interne ; que leur violation peut être invoquée à l'appui d'un recours devant le juge [interne] » (CFJ/CAV, 08 juin 1971, arrêt n $\left.{ }^{\circ} 63 / \mathrm{A}\right)$. Ce qui constitue une reconnaissance du juge interne comme serviteur du droit d'origine externe. Mission consistant donc principalement à interpréter et à appliquer ce droit. Seulement, bien que le principe en matière d'application soit maintenu intact en droit communautaire, comme nous l'avons vu cidessus, il est cependant limité en matière d'interprétation où la charge revient exclusivement à la juridiction communautaire. 
Pour des raisons de sécurité de l'ordre juridique communautaire et pour assurer l'application uniforme du droit issu des traités communautaires et des actes pris par ses organes, la quasi majorité des organisations d'intégration ont institué une coopération judiciaire entre les juridictions nationales et une juridiction communautaire. Cette coopération repose sur l'interprétation du droit communautaire. Ainsi, il est reconnu que la juridiction communautaire statue à titre préjudiciel sur l'interprétation du Traité constitutif et des Textes subséquents, sur la légalité et l'interprétation des Statuts et des Actes des Organes de la Communauté, quand une juridiction nationale ou un organisme à fonction juridictionnelle est appelée à en connaître à l'occasion d'un litige. "En outre, chaque fois qu'une juridiction nationale ou un organisme à fonction juridictionnelle saisi de questions de droit ci-dessus doit statuer en dernier ressort, il est tenu de saisir préalablement la [juridiction communautaire]. Cette saisine devient facultative lorsque la juridiction nationale ou l'organisme à fonction juridictionnelle doit statuer à charge d'appel » (Article 26 de la convention du 30 Janvier 2009, régissant la Cour de justice de la CEMAC).A travers ceci, le système juridique communautaire organise une cogestion inévitable entre le juge national et le juge communautaire (KENFACK (J.), 2005), qui doivent nécessairement coopérer pour l'application effective du droit communautaire (TCHUINTE (J.), 2001).

En dépit des apparences, qui semblent faire montre d'un dessaisissement du juge interne de son pouvoir ou de sa soumission au juge communautaire, il ressort à la réalité que le rôle accordé au juge interne dans cette procédure de renvoi préjudiciel est primordial. En effet, son action est ici plus étendue, puisqu'il dispose d'un large pouvoir d'appréciation quant aux conditions de mise en œuvre de la question préjudicielle devant la Cour de justice communautaire, en ce sens que « la saisine de la Cour se fait par le juge lui-même, qui détient l'exclusivité de cette initiative. Il décidera d'y procéder, après une série d'interprétations, dont il a été maître jusqu'à ce jour » (BATAILLER (F.), 1963), p. 757.

En tout état de cause, le juge interne reste et demeure la cheville ouvrière de la coopération judiciaire instaurée par la technique du renvoi préjudiciel, qui est la voie royale de l'effectivité de la primauté du droit communautaire, tout simplement parce qu'aucun renvoi n'est possible s'il n'est conduit par le juge national. Le juge communautaire ne pouvant s'auto saisir et les particuliers ne pouvant le saisir, le détenteur du vrai pouvoir de mise en œuvre du droit communautaire n'est autre que le juge national, car comme soutient Joël RIDEAU, le rôle des « juges nationaux est d'autant plus fondamental que leur mission communautaire est essentielle dans le contrôle de l'application du droit communautaire. Ils sont chargés d'assurer le respect de ce droit par les particuliers et surtout par les autorités 
nationales. Ils sont, par l'exercice de leurs pouvoirs, les garants de la primauté du droit communautaire. Cette tâche appartient aussi à la Cour de justice des Communautés (...), saisie par la Commission ou par un autre État membre, dans le cadre de la procédure en constatation des manquements. L'importance des procédures nationales est cependant fondamentale parce qu'elles seules permettent aux particuliers de soumettre à un juge les méconnaissances du droit communautaire par les autorités nationales. » (RIDEAU (J.), 1972, p.883)

C'est donc dire que le juge national est véritablement le juge communautaire de droit commun, non seulement du fait que c'est sur lui que repose la charge de protéger les droits des particuliers, mais aussi parce qu'il doit veiller sur la primauté du droit communautaire en diligentant en cas de besoin les questions préjudicielles auprès de la Cour de justice communautaire. Mission qui contribue à l'uniformité d'application du droit communautaire.

\section{CONCLUSION}

L'épineuse question de la primauté du droit communautaire est de loin la plus critique des débats sur les rapports entre les systèmes juridiques. Parce que l'ordre juridique communautaire ne saurait se concevoir autrement que primant sur les ordres de ses membres. Sa nature intégratrice et la force de pénétration de ses normes, mues par l'effet direct et immédiat, ne peut se contenter d'une simple cohabitation avec le droit national, surtout constitutionnel. Cependant les allants souverainistes des constitutions africaines digèrent mal ou pas l'idée d'une deuxième place dans la hiérarchie des normes. En tant que distributrices des compétences, les constitutions se sont taillées la part du lion en conservant et en proclamant leur suprématie sur toutes les normes validées par elles. Conséquence, ce chauvinisme constitutionnel a abouti à une schizophrénie normativo-institutionnelle des ordres juridiques constitutionnels des États, où l'on assiste à un dépassement du critère organique et formel de la hiérarchie des normes et des fonctions de l'État (BECHILLON (D.), 1996. p.5) La pyramide kelsenienne peine à rester debout (TROPER (M.), 1978 pp 1523-1536, AMSELEK (P.), 1978, pp. 519 ; AUTEXIER (C.), 1982. pp. 337-372), du moins si elle est toujours debout, 1'ordre des normes porte de plus en plus au désordre normatif (NICOLAS (V.), 1994, pp.35-48). Est-il encore pertinent de parler de la hiérarchie des normes de nos jours lorsqu'on constate avec ce qui précède que, la norme communautaire peut, par le jeu de sa mise en œuvre, se confondre à (ou se fondre dans) la norme constitutionnelle, législative et réglementaire sans que sa qualification intrinsèque ait subi une quelconque modification ? Par ce fait, l'on peut dire que ce droit est d'une flexibilité (CARBONIER (J.), 2008) qui le met en dehors des stéréotypes classiques de la hiérarchie des normes. Par 
conséquent, il est un droit « hors hiérarchie » (NGAH (A. M.), 2018). Ainsi, il est indéniable qu'il est plus que jamais temps de « repenser les rapports entre ordres juridiques (BONNET (B.), 2013). La recherche de solutions dans le cadre de rapports de systèmes permet de sortir des apories inhérentes à la recherche systématique de la hiérarchie des normes en permettant le maintien d'une harmonie indispensable au bon fonctionnement d'un ensemble constitué de plusieurs systèmes sédimentés les uns aux autres. (BONNET (B.), 2013). Cet idéal justifie- t-il l'union libre qui caractérise les rapports ordres constitutionnels des États et ordre juridique communautaire tel que cela est perçu en matière d'application immédiate de ce dernier?

\section{References:}

1. AMSELEK (P.), «Réflexions critiques autour de la conception kelsénienne de l'ordre juridique », R.D.P., 1978, 5-19

2. AUTEXIER (C.), «L'hétérogénéité du droit communautaire dérivé », Revue internationale de droit comparé. Vol. 34 №2, Avril-juin 1982.

3. BATAILLER (F.), " Le juge interne et le Droit communautaire », AFDI, Vol.9, 1963, p.

4. BECHILLON (D.), Hiérarchie des normes et hiérarchie des fonctions normatives de l'État, Economica, Paris, 1996

5. BÉLIGH NABLI « L'Union des États et les États de l'Union», Pouvoir $\mathrm{n}^{\circ} 121,2007$

6. BERRAMDANE (A.), La hiérarchie des droits: Droits internes et droit européen et international, L'Harmattan, Paris, 2002

7. BONNET (B.), Repenser les rapports entre ordres juridiques, Paris, Lextenso, 2013

8. BOUSSETTA (S.), « L'article 54 de la Constitution française : Contrôle de hiérarchie des normes ou contrôle de compatibilité des normes ? », www.droitconstitutionnel.org, 2017

9. BRIBOSIA (H.), «Applicabilité directe et primauté des traités internationaux et du droit communautaire- Réflexions générales sur le point de vue de l'ordre juridique belge», RBDI, 1996

10. CAMBY (J.P.), "Le droit communautaire est-il soluble dans la Constitution ?", RDP, 2004

11. CANIVET (G.), « Droit communautaire et l'office du juge national », Droit et Société, 1992

12. CARBONIER (J.), Flexible droit : pour une sociologie du droit sans rigueur, $10^{\mathrm{e}}$ édition, Paris, LGDJ, 2008

13. DE BERRANGER (T.), Constitutions nationales et construction communautaire, LGDJ, Paris, 1995

14. DE VISSCHER (P.), « Les tendances internationales des constitutions modernes », RCADI, 1951 
15. DUBOUT (E.), «De la primauté “'imposée" à la primauté "consentie" : Les incidences de l'inscription du principe de primauté dans le traité établissant une Constitution pour l'Europe »in IVe congrès de droit constitutionnel de l'AFDC, Montpellier, 9, 10 et 11 juin 2005

16. FIPA NGUEPJO (J.), Le rôle des juridictions supranationales de la Cemanc et de l'OHADA dans l'intégration des droits communautaires par les Etats membres, Thèse, Université de Paris 2, 2011

17. GNIMPIEBA TONNANG (E.),NGUENA DJOUFACK (A.L.), «Influence du droit communautaire de la CEMAC sur le domaine de compétence du juge administratif camerounais », www.legavox.fr, 2014)

18. HECQUARD-THERON (M.), « La notion d'État en droit communautaire », RTDE, $1990, \mathrm{n}^{\circ} 4$, p. 693-711

19. KAMTO (M.), « La volonté de l'Etat en droit international », RCADI, Nijhoff, 2007

20. KENFACK (J.), «Le juge camerounais à l'épreuve du droit communautaire et de l'intégration économique », in Juridis périodique, ${ }^{\circ} 63$, Juillet-août-septembre 2005

21. LUCHAIRE (F.), in La Constitution et l'Europe, Paris, Montchrestien, 1992

22. NDJIMBA (K.F.), L'internationalisation des constitutions des Etats en crise : réflexion sur les rapports entre Droit international et Droit constitutionnel, Thèse, Université de Nancy 2, 2011

23. NGAH (A. M.), L'ordre juridique communautaire et les constitutions des Etats membres de la CEMAC et de la CEDEAO, Thèse, Université de Yaoundé II, 2018

24. NGUELE ABADA (M.), «Le contrôle de constitutionnalité des lois au Cameroun », in La Constitution camerounaise du 18 janvier 1996 : Bilan et perspectives, ONDOUA (A.) (dir.), Afrédit, 2007

25. NICOLAS (V.), " Le désordre normatif », pouvoir n ${ }^{\circ} 69,1994$

26. ONDOUA (A.), «Le droit international dans la constitution camerounaise ", in Le Cameroun et le droit international, ATANGANA AMOUGOU (J.L.) (dir.), Paris, Pedone, 2014

27. ONDOUA (A.), Étude des rapports entre le droit communautaire et la constitution en France, L'harmattan, Paris, 2001

28. OUEDRAOGO (S.M.), La lutte contre la fraude à la constitution en Afrique noire francophone, Thèse, Université Montesquieu-Bordeau IV, 2011

29. PELLET (A.), «Les fondements internationaux du droit communautaire ». Academy of European Law, Firenze, Recueil des cours, 1994 
30. PESCATORE (P.), Le droit de l'intégration, Bruxelles, Bruylant, coll. « Droit de l'Union européenne », 2005 ;

31. PFERSMANN (O.), «La primauté : double, partiellement directe, organiquement indéterminée, provisoirement fermée ", Cahiers $d u$ Conseil constitutionnel $n^{\circ} 18$ (Dossier : Constitution et Europe) -juillet 2005

32. PICOD (F.), «La normativité du droit communautaire », Cahiers $d u$ Conseil constitutionnel, n²1, Dossier spécial "La Normativité ", 2007 ;

33. RIDEAU (J.), « Le rôle des États membres dans l'application du droit communautaire», AFDI, 1972

34. RIDEAU (J.), "Constitution et droit international dans les États membres des Communautés européennes », RFDC, 1990, p.270.).

35. SAWADOGO (M.F.), " Le juge national et le droit communautaire», actes $d u$ Colloque de Ouagadougou, 24-26 juin 2003, p.77

36. SUBTIL (J.B.), Le Monde, 30 novembre 1990

37. TCHUINTE (J.), L'application effective du droit communautaire en Afrique Centrale, Thèse, Université Cergy Pontoise, 2001

38. TROPER (M.), «La pyramide est toujours debout! Réponse à Paul AMSELEK », RDP, 1978 